\title{
Does COVID-19 Worsen the Semen Parameters? Early Results of a Tertiary Healthcare Center
}

\author{
Erdem Koça Buğra Bilge Keseroğlu ${ }^{b}$ \\ aDepartment of Urology, Ankara Yıldırım Beyazıt University, School of Medicine, Ankara State Hospital, Ankara,

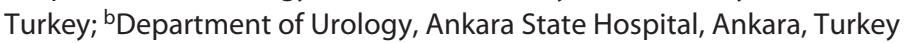

\section{Keywords}

Semen analyses $\cdot$ Testosterone $\cdot$ Severe acute respiratory syndrome coronavirus 2 . Coronavirus disease 2019 .

Infertility

\begin{abstract}
Introduction: The coronavirus disease 2019 (COVID-19) is a global pandemic which may affect multiple organs and systems including testes and disrupt the gonadal functions. The current study aimed to evaluate the effect of COVID-19 on the semen parameters and sex-related hormone levels in infertile men. Methods: The study included 21 patients who were evaluated in Ankara City Hospital, Andrology Clinic, for male infertility and have had the diagnosis of COVID-19. All the patients were evaluated in terms of semen parameters. The follicle-stimulating hormone, luteinizing hormone, and testosterone $(\mathrm{T})$ levels were also evaluated in 8 of the patients. The results were presented through 2 dependent group analyses, based on the data of the patients collected before and after the diagnosis of COVID-19. Results: None of the patients needed to be hospitalized at any time through the course of COVID-19. There was a significant decrease in semen volume, percentage of total motility, percentage of
\end{abstract}

progressive motility, and normal sperm morphology after COVID-19 (3 [1-8] vs. 2.5 [1.5-5], $p=0.005 ; 48.6 \pm 22.1$ vs. 34.7 $\pm 20.7, p=0.001 ; 35.1 \pm 21.7$ vs. $21.8 \pm 15.9, p<0.001 ; 6$ [3-24] vs. 5 [3-18], $p=0.015$; respectively). There was also a significant decline in $\mathrm{T}$ level of the patients after the diagnosis of COVID-19 (350.1 \pm 115.5 vs. $289.8 \pm 103.3, p=0.009)$. Conclusion: COVID-19 may have unfavorable effects on the gonadal functions and may lead to further deterioration of the semen parameters in infertile men, which should be considered through the evaluation for infertility.

(c) 2021 S. Karger AG, Basel

\section{Introduction}

The novel severe acute respiratory syndrome coronavirus 2 (SARS-CoV-2) is a member of the Coronaviridae family and the infectious agent of the coronavirus disease 2019 (COVID-19). COVID-19 was first diagnosed in Wuhan, China, in December 2019 and has since become a global pandemic [1]. The COVID-19 primarily affects the respiratory system, as well as other systems such as the gastrointestinal system, cardiovascular system, and hematological system [2].
Correspondence to:

Erdem Koç, drerdemkoc@gmail.com 
There are a number of studies presenting that COVID-19 may affect the testes [3-5] and male genital tract which may in turn disrupt the gonadal functions $[6,7]$. The destructive effects of the disease may be the result of either the direct cytopathic effects of SARS-CoV-2 on the target cells or the indirect damage through the inflammatory response against the virus [3]. Cell entry of SARS$\mathrm{CoV}-2$ depends on the binding of the viral spike (S) proteins to cellular receptors and $S$ protein priming by host cell proteases. Angiotensin-converting enzyme-2 (ACE2) receptors are thought to play a key role as to be a passage for SARS-CoV-2 to invade the target cells and activate the serine protease TMPRSS2 for priming the S protein, which defines the release of the $\mathrm{S} 2$ subunit allowing the fusion of viral and cellular membranes [8-12]. Spermatogonia, Leydig cells, Sertoli cells, prostate tissue, and seminiferous tubules were also shown to express ACE-2 receptors, suggesting that the COVID-19 may adversely affect the male reproductive system $[6,13,14]$.

Previous studies also reported that SARS-CoV-2 may cross the blood-brain barrier and infect the neurons and glial cells through the ACE- 2 receptors and trigger some inflammatory processes in neural tissues including the hypothalamus and pituitary gland. On the other hand, the possible influence of SARS-CoV-2 infection on the hypothalamic-pituitary-adrenal axis has been speculated, and the mechanisms through which COVID-19 disrupts the functioning of the hypothalamic-pituitary-gonadal axis are unclear $[15,16]$.

In this current study, we aimed to elucidate the change in semen parameters of the infertile men who had been infected with SARS-CoV-2, through the comparison of the spermiogram results which were reported before and after the diagnosis of COVID-19. We also aimed to investigate the impact of COVID-19 on the sex-related hormone levels such as follicle-stimulating hormone (FSH), luteinizing hormone $(\mathrm{LH})$, and testosterone $(\mathrm{T})$.

\section{Materials and Methods}

The current study was conducted in a tertiary healthcare center, Ankara City Hospital, Turkey. The study included 21 patients who applied to the Andrology Clinic for male infertility and have had the semen analyses reported as before and after the diagnosis of COVID-19. The study protocol was approved by the Institutional Review Board of Ankara City Hospital, after the approval of the Ethics Committee of the Ministry of Health, Republic of Turkey (\#E1-20-1237), and approved by the US National Library of Medicine, National Institutes of Health (\#NCT04643522).

Patients' age, smoking status, BMI, medical history, and the presence of varicocele at physical examination were recorded. Be- sides the semen parameters, 8 of the patients were also analyzed in terms of sex-related hormone levels (FSH, LH, and T). The serum samples were obtained from venous blood taken at 8:00-11:00 a.m., on the same day with the semen sampling, and were used for hormone level measurements. The patients' data which were collected before and after the diagnosis of COVID-19 were compared through 2 dependent group analyses.

The diagnosis of COVID-19 has been established according to the detection of SARS-CoV-2 in the samples taken from the nasopharynx with a cotton swab and were examined through RT-PCR, in the Microbiology Laboratory. None of the patients were at the active phase of the COVID-19 at the time of the semen sampling.

All of the semen samples were examined in the Andrology Laboratory, by 2 experienced biologists. The samples were processed according to WHO guidelines (2010, 5th edition) through the routine semen analysis. From the 2 consecutive semen samples, the one with the better results was included in data analysis, for both the dependent groups. Semen samples were collected through masturbation after 3-5 days of sexual abstinence and examined following $30-60 \mathrm{~min}$ of liquefaction at $37^{\circ} \mathrm{C}$. The samples were manually evaluated in terms of volume, liquefaction time, and $\mathrm{pH}$ value and then assessed by using optical microscopy to report the concentration, motility (total, progressive, nonprogressive, and immotile), and morphology.

\section{Statistical Analysis}

Statistical analyses were performed by using the SPSS for Windows version 22.0 (SPSS Inc., Chicago, IL, USA) software package. The normality of the data was assessed via the Shapiro-Wilk test. Paired-samples $t$ test was used in analyses of normally distributed variables, and the results were presented as mean \pm SD. McNemar $\chi^{2}$ test and Wilcoxon signed rank test were used if the variables were not normally distributed, and the data were presented as $n$ (\%) and median (min-max), respectively. With the confidence interval of $95 \%$, a $p$ value $<0.05$ was considered as statistically significant.

Power analysis was performed by using G-power software (Gpower v3.1.9.2; Universitat Kiel, Kiel, Germany). In the post hoc power analysis, the power of the study was found to be 0.80 with a 0.65 effect size and 0.05 error rate, for dependent group analyses of the semen analysis data of 21 patients with COVID-19.

\section{Results}

The data of overall 25 patients who applied to Ankara City Hospital, Andrology Clinic, for infertility evaluation and who have had the spermiogram results reported before and after the diagnosis of COVID-19 were reviewed. Four of the patients were found to have azoospermia. Due to the technique difficulties to compare the change at semen parameters in case of azoospermia as before and after results, these 4 patients were excluded. Therefore, the study included 21 patients in final analyses. 
Table 1. Demographic features and clinical characteristics of the patients

Patients, $N$

Age, mean \pm SD, years

21

$\mathrm{BMI}$, mean $\pm \mathrm{SD}, \mathrm{kg} / \mathrm{m}^{2}$

Smoking history (yes/no), $n$ (\%)

Time between the diagnosis of COVID-19 and semen analyses performed after the COVID-19, median (min-max), days

Time between the diagnosis of COVID-19 and semen analyses performed before the COVID-19, median (min-max), days

Clinical varicocele presence (yes/no), $n(\%)$

$32 \pm 6.30$

$25.62 \pm 2.12$

$6 / 21(28.5)$

$51(37-89)$

$116(72-238)$

$4 / 21(19)$

COVID-19, coronavirus disease 2019; SD, standard deviation; min-max, minimum-maximum.

Table 2. Semen analysis results of the patients before and after the diagnosis of COVID-19

\begin{tabular}{|c|c|c|c|}
\hline Semen parameters & $\begin{array}{l}\text { Before COVID-19 } \\
\text { diagnosis }\end{array}$ & $\begin{array}{l}\text { After COVID-19 } \\
\text { diagnosis }\end{array}$ & $p$ value \\
\hline Duration of sexual abstinence, median (min-max), days & $4(3-5)$ & $4(4-4)$ & 1.00 \\
\hline Volume, median (min-max), mL & $3(1-8)$ & $2.5(1.5-5)$ & $0.005^{*}$ \\
\hline Sperm concentration, median $(\min -\max ), \times 10^{6} / \mathrm{mL}$ & $42(2-148)$ & $35.9(4-126.5)$ & 0.689 \\
\hline Total sperm number $\left(\times 10^{6}\right.$ per ejaculate), median (min-max) & $145(6-788)$ & $96.9(13.4-603.5)$ & 0.654 \\
\hline Total motility, mean \pm SD, $\%$ & $48.6 \pm 22.1$ & $34.7 \pm 20.7$ & $0.001^{*}$ \\
\hline Progressive motility, mean $\pm \mathrm{SD}, \%$ & $35.1 \pm 21.7$ & $21.8 \pm 15.9$ & $<0.001^{*}$ \\
\hline Nonprogressive motility, mean $\pm \mathrm{SD}, \%$ & $12.9 \pm 6.7$ & $12.9 \pm 7.6$ & 1.00 \\
\hline Immotility, mean \pm SD, $\%$ & $51.4 \pm 22.1$ & $65.3 \pm 20.7$ & $0.001^{*}$ \\
\hline Sperm morphology (\% of normal forms), median (min-max) & $6(3-24)$ & $5(3-18)$ & $0.015^{*}$ \\
\hline
\end{tabular}

COVID-19, coronavirus disease 2019; SD, standard deviation; min-max, minimum-maximum. ${ }^{*} p$ value $<0.05$ was considered as statistically significant.

Table 3. Sex-related hormone levels before and after the diagnosis of COVID-19

\begin{tabular}{llll}
\hline Sex-related hormones & Before COVID-19 diagnosis & After COVID-19 diagnosis & $p$ value \\
\hline FSH, mean \pm SD, U/L & $4.6 \pm 1.8$ & $4.7 \pm 1.6$ & 0.692 \\
LH, mean \pm SD, U/L & $3 \pm 1.2$ & $3.1 \pm 1.3$ & 0.491 \\
T, mean \pm SD, ng/dL & $350.1 \pm 115.5$ & $289.8 \pm 103.3$ & $0.009^{*}$ \\
\hline
\end{tabular}

COVID-19, coronavirus disease 2019; FSH, follicle-stimulating hormone; LH, luteinizing hormone; T, testosterone; SD, standard deviation. ${ }^{*} p$ value $<0.05$ was considered as statistically significant.

The age of the patients ranged between 23 and 42 years. The demographic features and clinical characteristics of the patients are summarized in Table 1.

The patients were detected to have complaints of fever $(n=2)$, dyspnea $(n=1)$, cough $(n=6)$, myalgia $(n=13)$, headache $(n=14)$, weakness $(n=13)$, diarrhea $(n=3)$, anorexia $(n=8)$, and sore throat $(n=7)$ at the time of the diagnosis of COVID-19. None of the patients had any clinical finding suggesting orchitis. None of the patients needed to be hospitalized at any time through the course of the COVID-19; however, 3 patients used hydroxychloroquine sulfate $2 \times 200 \mathrm{mg}$ per day for 5 days, and 2 pa- 
tients used favipiravir $2 \times 1,600 \mathrm{mg}$ loading dose at the first day and $2 \times 600 \mathrm{mg}$ per day maintenance dose for 4 days in terms of the medications.

The semen volume, percentage of total motility, percentage of progressive motility, and normal sperm morphology were found as significantly decreased after COVID-19 $(p=0.005, p=0.001, p<0.001$, and $p=0.015$, respectively). Besides, the percentage of immotile sperms was observed as significantly increased after COVID-19 ( $p=0.001)$ (Table 2). In terms of sex-related hormone levels, there was a significant decrease in T level after the diagnosis of COVID-19 ( $p=0.009)$ (Table 3).

\section{Discussion}

The current study revealed that COVID-19 significantly worsens the semen parameters. Serum T level was also observed as declined after the diagnosis of COVID-19. To the best of our knowledge, this study is the first in the literature to assess the impact of COVID-19 on semen parameters and sex-related hormone levels, through the dependent group analyses of the data collected before and after the diagnosis of COVID-19.

In a study conducted by Holtman et al. [17], the semen analysis results of 18 patients infected with SARS-CoV-2 were compared to the results of 14 healthy males. They reported that sperm concentration and motility were significantly higher in the control group [17]. The current study appears to be consistent with this previous study in terms of the significant decrease in sperm motility after the diagnosis of COVID-19. Even though the sperm concentration was observed as decreased after the diagnosis of COVID-19 in the current study, this difference was not statistically significant.

In another study, Ma et al. [18] compared the data of 119 patients with COVID-19 to the control group including 273 healthy men. They did not report any significant difference in terms of T and FSH levels between the 2 groups, while the LH level was significantly higher, and the $\mathrm{T} / \mathrm{LH}$ ratio was significantly decreased in patients with the diagnosis of COVID-19 [18]. On the other hand, the current study revealed a significant decrease in $\mathrm{T} \mathrm{lev-}$ els of the patients after the diagnosis of COVID-19. Even though the sex-related hormone levels were analyzed in a low number of patients, the findings of the current study are important to present the dependent group analysis results of a hormone, such as $\mathrm{T}$, which has a wide variation throughout the population [19]. Additionally, Ma et al. [18] also analyzed the semen samples of 12 patients after 56-109 days from the diagnosis of COVID-19 and reported that 4 patients had low sperm motility. Besides, only 3 of the patients had the spermiogram results before and after the diagnosis of COVID-19, in which the sperm motility was reported as decreased in 2 of them [18]. However, they did not have a control group, and their patient number was very low in order to analyze the change in spermiogram results.

In the autopsy series reported by Xu et al. [20], SARS$\mathrm{CoV}$, the causative agent of SARS disease, was shown to be related with the development of orchitis. In histopathological investigations, they reported inflammatory infiltrates, mainly in the seminiferous tubules. Even though they could not isolate the viral genomic materials of SARS-CoV at in situ hybridization of the testicular tissue specimens, they interpreted the orchitis as the result of virus-related inflammatory and immunological reactions [20]. There are also a number of autopsy series which revealed similar destructions in histopathological examination of the male reproductive system in infected patients with another Coronaviridae family member, SARSCoV-2. In a study conducted by Bian and team [3], the authors reported that SARS-CoV-2 was isolated from the testes in their autopsy series. In addition, they reported various degrees of injury in testicular cells and reduction in the number of spermatogenic cells [3]. In another autopsy series, Achua et al. [4] also detected SARS-CoV-2 positivity in the testes, and they also detected impaired spermatogenesis. Through the insight of the literature, we may argue that the inflammatory destruction of COVID-19 may be the result of the general systemic inflammatory effect of the virus or the result of the viral destruction directly targeting the testes. SARS-CoV-2 may affect the Leydig cells through the ACE- 2 receptors which may in turn decrease the T level and affect the spermatogenesis and result in a decline in semen parameters [21].

The study conducted by Fan et al. [6] elucidated that SARS-CoV-2 mostly uses ACE- 2 receptors to invade the host cells. ACE-2 receptors were shown to be abundant in spermatogonia, Leydig cells, and Sertoli cells [13] and were also detected in prostate tissue and seminal vesicles [22]. Most of the seminal fluid was excreted by the prostate and seminal vesicles [23]. With regard to the data, the significant decrease in semen volume observed at the current study may be explained by the impact of COVID-19 on the prostatic cells and seminal vesicles.

In the study conducted by Achua et al. [4], there was an important finding that spermatogenesis was more prominently affected by COVID-19 in cases with increased ACE-2 expression. As another interesting find- 
ing, the ACE- 2 receptors have been previously shown to be expressed in higher numbers through the reproductive age [24]. Therefore, we may also argue that the testicular damage should be more significant during the reproductive period. Considering that $60 \%$ of the SARS-CoV-2-infected population was detected to be male gender and approximately $60 \%$ of the males have been at the reproductive age, it raises the concerns whether SARS-CoV-2 may affect the male reproductive functions as a globally increasing problem [25]. In this aspect, the male population in the reproductive period with a history of COVID-19, especially the male patients with a previously established diagnosis of infertility, should be carefully evaluated in terms of gonadal functions.

The current study has a number of limitations regarding its retrospective nature. The number of the participants evaluated for hormone levels was limited; however, it was not possible to plan the evaluation of all patients in terms of any parameter such as hormone levels due to the retrospective design of the study. Even though this study has been conducted in a high-volume tertiary health care center, the total number of the patients was also restricted probably related with the difficulty in transportation of patients to the hospital during the pandemic period.

The life cycle of the spermatozoa has been reported to be approximately 74 days [26]. Considering the data, disturbed semen characteristics which were presented in this current study may be accepted as the early results of the impact of COVID-19 on semen parameters. Further studies may be designed to better understand the longterm effects of COVID-19 on spermiogram results.

\section{Conclusion}

The current study revealed important findings regarding the significant unfavorable effects of COVID-19 on the semen parameters. Serum T level was shown to be significantly decreased after the diagnosis of COVID-19. The clinicians should be cautious that COVID-19 may lead to further deterioration of the semen parameters which may be crucial in evaluation of infertile men.

\section{Acknowledgments}

We sincerely thank the 2 experienced biologists Ümit Özgen and Zehra Yiğiter, Andrology Laboratory, Ankara City Hospital, for their contributions in examination of semen samples.

\section{Statement of Ethics}

The research was conducted in accordance with the World Medical Association Declaration of Helsinki. Written informed consent was taken from all participants. The study protocol was approved by the Institutional Review Board of Ankara City Hospital, after the approval of the Ethics Committee of the Ministry of Health, Republic of Turkey (\#E1-20-1237), and approved by the US National Library of Medicine, National Institutes of Health (\#NCT04643522).

\section{Conflict of Interest Statement}

The authors declare no conflicts of interest to disclose.

\section{Funding Sources}

There are no funding sources to declare.

\section{Author Contributions}

Koç E. contributed to the study conceptualization, study design, data collection, study writing, review, and editing. Keseroğlu B.B. contributed to study design, data collection, and statistical analyses.

\section{References}

1 Coronavirus Disease (COVID-19) Pandemic. World Health Organization. Geneva. 2020 Apr 9 [cited 2020 Nov 21]. Available from: https: //www.who.int/emergencies/diseases/ novel-coronavirus-2019.

2 Gupta A, Madhavan MV, Sehgal K, Nair N, Mahajan S, Sehrawat TS, et al. Extrapulmonary manifestations of COVID-19. Nat Med. 2020;26(7):1017-32.
3 Bian XW, Team TCP. Autopsy of COVID-19 patients in China. Natl Sci Rev. 2020;7(9): $1414-8$.

4 Achua JK, Chu KY, Ibrahim E, Khodamoradi K, Delma KS, Iakymenko OA, et al. Histopathology and ultrastructural findings of fatal COVID-19 infections on testis. World J Mens Health. 2020;38:e56.
5 Yang M, Chen S, Huang B, Zhong JM, Su H Chen YJ, et al. Pathological findings in the testes of COVID-19 patients: clinical implications. Eur Urol Focus. 2020;6(5):1124-9.

6 Fan C, Li K, Ding Y, Lu W, Wang J. ACE2 expression in kidney and testis may cause kidney and testis damage after 2019-nCoV infection. medRxiv. 
7 Temiz MZ, Dincer MM, Hacibey I, Yazar RO, Celik C, Kucuk SH, et al. Investigation of SARS-CoV-2 in semen samples and the effects of COVID-19 on male sexual health by using semen analysis and serum male hormone profile: a cross-sectional, pilot study. Andrologia. 2021 Mar;53(2):e13912.

8 Hoffmann M, Kleine-Weber H, Schroeder S, Krüger N, Herrler T, Erichsen S, et al. SARSCoV-2 cell entry depends on ACE2 and TMPRSS2 and is blocked by a clinically proven protease inhibitor. Cell. 2020;181(2):271-e8.

9 Kayaaslan B, Korukluoglu G, Hasanoglu I, Kalem AK, Eser F, Akinci E, et al. Investigation of SARS-CoV-2 in Semen of patients in the acute stage of COVID-19 infection. Urol Int. 2020;104(9-10):678-83.

10 Wiwanitkit V. SARS-CoV-2 in Semen. Urol Int. 2020;104(11-12):1000

11 Kayaaslan B, Korukluoglu G, Hasanoglu I, Kalem AK, Eser F, Akinci E, et al. Semen does not cause additional risk for SARS-CoV-2 transmission during sexual contact. Urol Int. 2020;104(11-12):1003-4.

12 Punjani N, Li PS, Alukal JP. Investigation of SARS-CoV-2 in Semen of patients in the acute stage of COVID-19 infection. Urol Int. 2020;104(11-12):1001-2.

13 Wang Z, Xu X. scRNA-seq profiling of human testes reveals the presence of the ACE2 receptor, a target for SARS-CoV-2 infection in spermatogonia, leydig and sertoli cells. Cells. 2020;9(4):920.

14 Huang C, Ji X, Zhou W, Huang Z, Peng X, Fan $\mathrm{L}$, et al. Coronavirus: a possible cause of reduced male fertility. Andrology. 2021;9(1):80-7.

15 Selvaraj K, Ravichandran S, Krishnan S, Radhakrishnan RK, Manickam N, Kandasamy M. Testicular atrophy and hypothalamic pathology in COVID-19: possibility of the incidence of male infertility and HPG axis abnormalities. Reprod Sci. 2021:1-8.

16 Ding Y, He LI, Zhang Q, Huang Z, Che X, Hou J, et al. Organ distribution of severe acute respiratory syndrome (SARS) associated coronavirus (SARS-CoV) in SARS patients: implications for pathogenesis and virus transmission pathways. J Pathol. 2004;203(2):62230.

17 Holtmann N, Edimiris P, Andree M, Doehmen C, Baston-Buest D, Adams O, et al. Assessment of SARS-CoV-2 in human semen-a cohort study. Fertil Steril. 2020;114(2):233-8.

18 Ma L, Xie W, Li D, Shi L, Ye G, Mao Y, et al. Evaluation of sex-related hormones and semen characteristics in reproductive-aged male COVID-19 patients. J Med Virol. 2021 Jan;93(1):456-62.

19 Lardone MC, Piottante A, Valdevenito R, Ebensperger M, Castro A. Histological and hor- monal testicular function in oligo/azoospermic infertile men. Andrologia. 2013;45(6): 379-85.

20 Xu J, Qi L, Chi X, Yang J, Wei X, Gong E, et al. Orchitis: a complication of severe acute respiratory syndrome (SARS). Biol Reprod. 2006;74(2):410-6.

21 Smith LB, Walker WH. The regulation of spermatogenesis by androgens. Semin Cell Dev Biol. 2014;30:2-13.

22 Song $H$, Seddighzadeh B, Cooperberg MR, Huang FW. Expression of ACE2, the SARS-CoV-2 receptor, and TMPRSS2 in prostate epithelial cells. bioRxiv. $2020 \mathrm{Apr}$ 25.

23 Roberts M, Jarvi K. Steps in the investigation and management of low semen volume in the infertile man. Can Urol Assoc J. 2009;3(6): 479-85.

24 Dutta S, Sengupta P. SARS-CoV-2 and male infertility: possible multifaceted pathology. Reprod Sci. 2020.

25 Guan WJ, Ni ZY, Hu Y, Liang WH, Ou CQ, $\mathrm{He} \mathrm{JX}$, et al. Clinical characteristics of coronavirus disease 2019 in China. N Engl J Med. 2020;382(18):1708-20.

26 Babakhanzadeh E, Nazari M, Ghasemifar S, Khodadadian A. Some of the factors involved in male infertility: a prospective review. Int J Gen Med. 2020;13:29-41. 